\title{
RADIO-PISTAGE DE GRANDES ALOSES ADULTES EN LOIRE
}

\author{
P. STEINBACH *, P. GUENEAU **, A. AUTUORO ***, D. BROUSSARD *** \\ Conseil Supérieur de la Pêche \\ 112 Faubourg de la Cueille - 86000 POJTIERS, France.
}

\begin{abstract}
RESUME
Dix grandes aloses (Alosa alosa) sont équipées d'émetteurs radio en Loire moyenne, à l'époque de leur migration anadrome. Le marquage a lieu 15 à $20 \mathrm{~km}$ en aval du seuil de St LAURENT-desEAUX, un des premiers obstacles sérieux rencontrés sur cet axe de migration.

Le suivi des poissons montre que la technique du radio-pistage peut être appliquée à cette espèce pourtant très sensible aux manipulations. Au-delà de ce test de fiabilité, les résultats confirment l'influence déterminante du facteur température sur la progression des aloses en rivière (inhibition au-dessous d'un seuil proche de $12^{\circ} \mathrm{C}$ ). On constate de même qu'une forte crue peut perturber momentanément leur remontée.
\end{abstract}

En l'absence de ces conditions hydrologiques défavorables, les données recueillies permettent également l'évaluation d'une vitesse moyenne de progression ( $20 \mathrm{~km} / \mathrm{j}$ ) sur parcours sans obstacle.

Enfin, la plupart des aloses marquées ayant été arrêtées par le seuil on observe leur comportement de repli et de regroupement en aval de celui-ci.

\section{SUMMARY}

\section{RADIO-TRACKING OF ATLANTIC SHADS IN THE RIVER LOIRE}

Ten Atlantic shads (Alosa alosa) were tagged with radio transmitters during their spawning migration in the middle River Loire. Radio-tagging occurred $15-20 \mathrm{~km}$ downstream of the weir of St LAURENT-des-EAUX, one of the first disturbing obstacles in the river to upstream migrants.

Radio telemetry can be successfully used to monitor the behavior of shad despite the apparent sensitivity of this species to handling. Radio-tracking results demonstrated that water temperature had a pronounced effect on shad swimming speed (migrations were inhibited at times when temperatures fell below 12 degrees $($ ). Peak flow rates in the river produced a similar effect on migratory behavior. In the absence of these adverse hydrological conditions, radio-tagged shad migrated upstream at an average rate of $20 \mathrm{~km} /$ day until they encountered the weir. Most of the tagged fish were impeded by this structure. The behavioral response (downstream movement, schooling) of shad blocked by the weir was noted.

\section{INTRODUCTION}

La grande alose (Alosa alosa) est un migrateur potamotoque dont l'aire de répartition englobe principalement les côtes est de l'Océan Atlantique. Dans les fleuves français (Adour, Garonne, Loire, Rhône), elle entame sa remontée entre février et avril, par l'arrivée de contingents où prédominent souvent les mâles, dans l'ensemble plus petits et d'un an plus jeunes que les femelles (DOUCHEMENT, 1981 ; CASSOU-LEINS et CASSOU-LEINS, 1981 ; DAUTREY et LARTIGUE, 1983 ; MENNESSON, 1984). Elle fraye généralement en mai-juin, l'optimum de température pour sa reproduction se situant entre 22 et $24^{\circ} \mathrm{C}$ (SPILLMANN, 1961). La ponte, fractionnée dans le temps et dans l'espace (LE CLERC, 1941 ; RAMEYE et al., 1976 ; CASSOU-LEINS et CASSOU-LEINS, 1981), a lieu sur gravières dans des zones de rapides (LE CLERC, 1941 ; C.T.G.R.E.F., 1975). Contrairement à l'Alose feinte (Alosa fallax), très peu d'individus survivraient à leur migration reproductrice (DOUCHEMENT, 1981.).

Sur le bassin de la Loire, les aires de reproduction, autrefois étendues et diversifiées, sont aujourd'hui mal répertoriées. En 1961, SPILLMANN situait encore leur limite amont sur l'Allier à hauteur d'Issoire. L'aménagement sur le fleuve et ses affluents de seuils et barrages constitue vraisemblablement l'une des principales causes de régression du stock en limitant ou bloquant l'accès à de nombreuses frayères potentielles.

\footnotetext{
* Ingénieur I.S.I.M.

* Ingénieur en Chef du G.R.E.F.

*** Garde-Chef C.S.P.

*** Garde-Pêche C.S.P.
} 
Beaucoup de questions restent en suspens concernant les parcours migratoires de la grande alose sur le bassin de la Loire, ainsi que son comportement face aux obstacles. Dans ce type de recherches, plusieurs études faisant appel au marquage radio ou ultrasonore ont été réalisées donnant des résultats satisfaisants sur l'espèce américaine (Alosa sapidissima) (LEGGETT et CARSCADDEN, 1971. LEGGETT et JONES, 1971 ; DODSON et LEGGETT, 1972, 1973, 1974 ; HYDROQUEBEC, 1982). Devant l'intérêt de telles recherches, le présent travail a pour but de tester les possibilités d'application de cette technique au cas de la grande alose. Le secteur géographique choisi pour ces investigations se situe sur le cours moyen de la Loire aux environs d'un des obstacles les plus marqués rencontrés par ce migrateur. II s'agit du seuil de SAINT LAURENT DES EAUX (Loir-et-Cher) ; cet ouvrage présente une hauteur moyenne de $1,35 \mathrm{~m}$ et n'est équipé d'aucun dispositif de franchissement.

\section{MATÉRIEL ET MÉthOdES}

\section{Marquage}

On utilise des émetteurs basses fréquences (40-41 $\mathrm{MHz}$ ), importés des Etats-Unis, présentant un diamètre de 12 à $14 \mathrm{~mm}$, une longueur de $5 \mathrm{~cm}$, un poids de $10 \mathrm{~g}$, pour une durée de vie de 40 à 50 jours et un rythme d'émission de 50 à 55 pulsations par minute.

Les poissons sont pêchés au filet barrage * ce qui présente l'avantage de ménager les sujets au moment de leur capture. De plus, afin de réduire la durée de l'opération, le marquage a lieu sur le bateau de pêche.

Dès sa sortie du carrelet, l'alose est déposée dans un bac circulaire, à l'intérieur duquel est immédiatement établi un courant tournant d'eau de Loire grâce à une pompe extérieure (cette circulation facilite le rétablissement de l'équilibre natatoire du poisson et lui assure une oxygénation optimale). Après une observation succincte concernant la taille, l'état physiologique (aspect extérieur) et la vivacité de l'alose, l'émetteur est poussé à l'intérieur de son œsophage au moyen d'un jeu de deux tubes (cf. schéma). L'extrémité de l'antenne d'émission (fil souple) esț simplement rabattue et calée à la commissure des maxillaires. Enfin, le sujet est relâché en Loire depuis l'embarcation, lorsque sa reprise d'équilibre dans le bac est jugée satisfaisante.

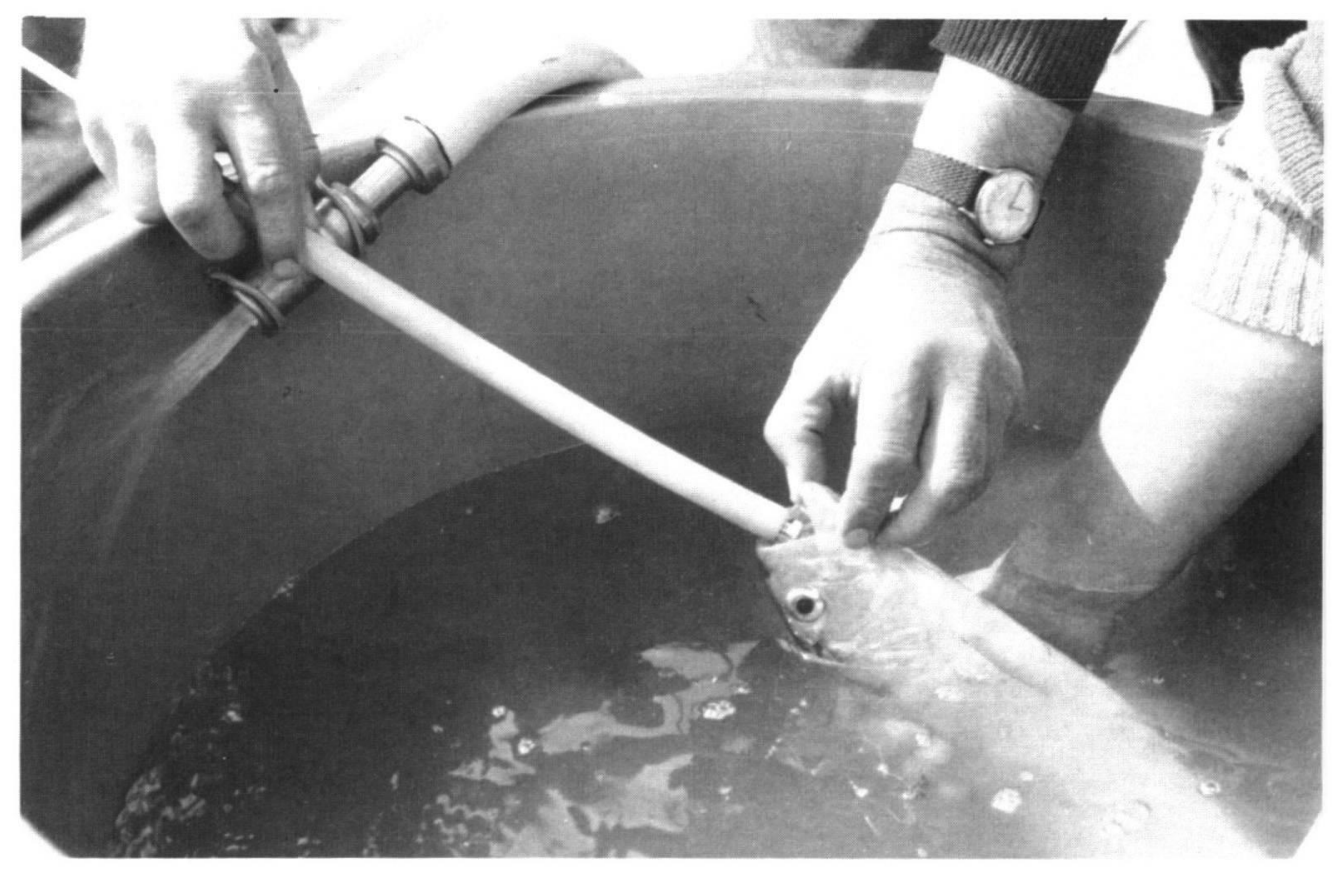

Photo 1 : Marquage d'une alose

Photo 1 : Shad tagging

* Filet perpendiculaire au courant, servant à guider les poissons migrateurs vers le carrelet d'un bateau équipé à cet effet (La Toue). 
Bull. Fr. Pêche Piscic. (1986) 302 : $106-117-108-$
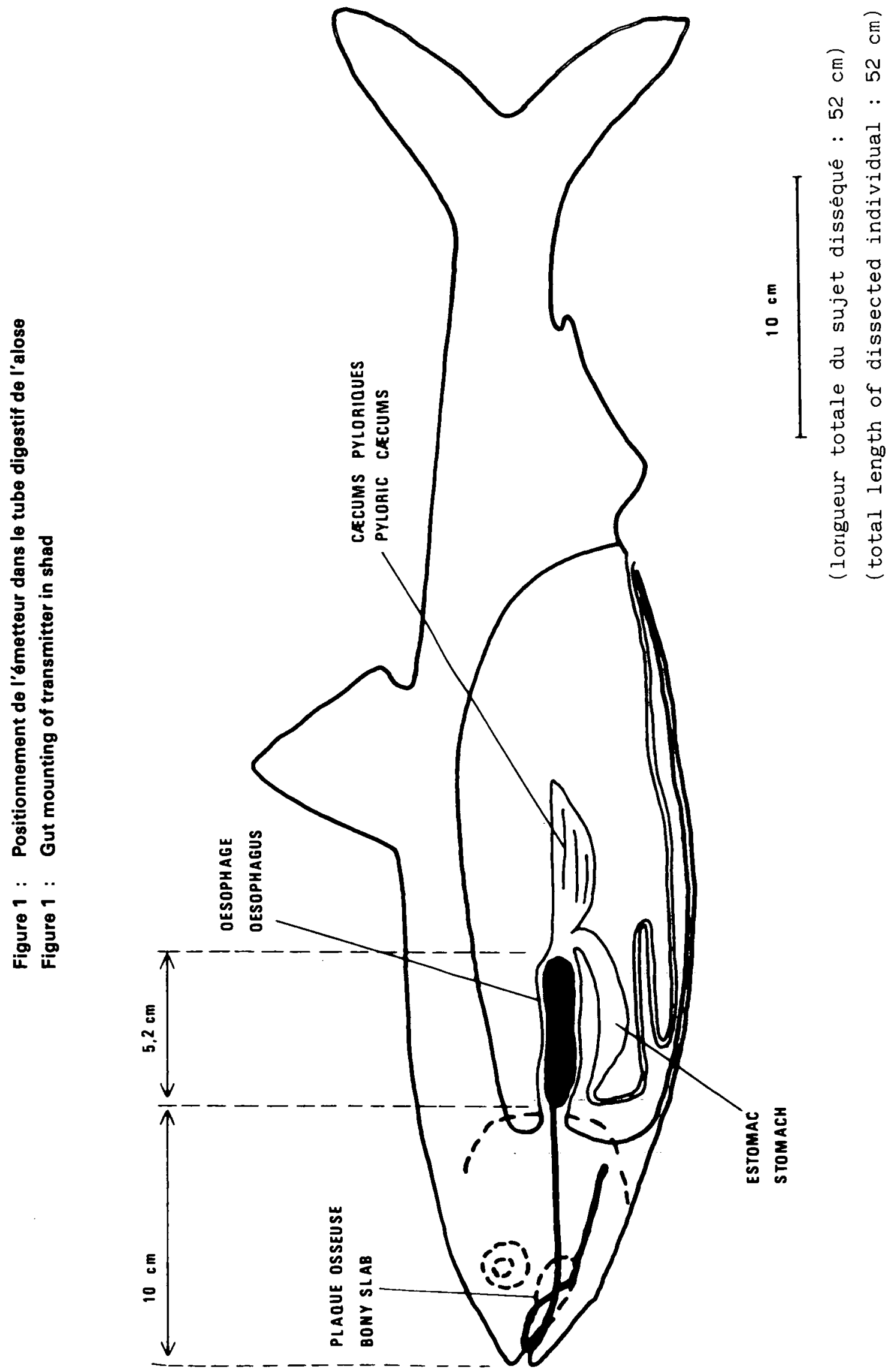
L'utilisation du bac à courant circulaire réduit le caractère limitant du temps écoulé entre la capture et le lâcher (P.G. HEISEY communication personnelle). Dans ces conditions les opérations décrites ci-dessus peuvent durer 4 a 5 minutes et s'effectuer sans précipitation. Par précaution celles-ci se déroulent loin en aval du seuil (15 a $20 \mathrm{~km}$ ); ainsi la faculté de récupération de chaque poisson peut être appréciée avant son arrivée devant l'obstacle.

Les marquages s'échelonnent entre le 25 avril et le 3 juin, époque à laquelle encore peu d'individus présentent des signes d'affaiblissement physiologique (maturation). Le principal critère de sélection parmi les poissons pêchés est la taille (compatibilité avec les dimensions des émetteurs utilisés). II est généralement préconisé d’éliminer les sujets dont l'équipement provoquerait un supplément pondéral de $2 \%$ dans l'air (poids du poisson $\leq 500 \mathrm{~g}$.). Dans le cas présent, cette limite n'est pas contraignante, mais afin de minimiser la gêne provoquée par l'ingestion de l'appareil, le choix des poissons porte sur des individus de $50-55 \mathrm{~cm}$ (soit $2 \mathrm{~kg}$ environ), taille légèrement supérieure à la moyenne rencontrée en Loire chez Alosa alosa. Au-delà, la sélection d'aloses de trop grande taille peut être défavorable à la rétention de l’émetteur dans l'cesophage (problème de régurgitation).

\section{Pistage}

Etant donné les objectifs du suivi (exploration méthodologique), aucun dispositif fixe de triangulation (localisation par recoupement goniométrique) n'est mis en place. Selon le niveau de précision exigé et l'étendue des recherches à entreprendre, les repérages sont effectués par voie routière. fluviale, aérienne ou simplement à pied depuis la berge. On emploie le plus souvent un récepteur monocanal muni d'une antenne boucle (directionnelle), cet équipement permettant de couvrir toute la largeur du fleuve avec une marge de sécurité satisfaisante. En se déplaçant le long de la berge les repérages au sol donnent avec précision la position du poisson projetée sur la rive. Par contre. à partir d'une trentaine de mètres son éloignement par rapport à la berge est déterminé de façon approximative.

Chaque alose est repérée plusieurs fois par jour, la fréquence des pointages variant suivant sa mobilité et son éloignement par rapport au seuil. Une station d'enregistrement munie d'un récepteur dix-canaux est mise en écoute permanente à l'amont immédiat de l'ouvrage. La cote de la Loire et la température de l'eau sont relevées quotidiennement pendant le suivi.

\section{RESULTATS}

\section{Evolution de la température et du débit de la Loire}

Un événement hydrologique a marqué le déroulement de la campagne d'étude. Il s'agit de la crue du mois de mai 1985. Cette montée des eaux a été brusque $(60 \mathrm{~cm} / \mathrm{jour}$ du 9 au 13 mai) et accompagnée d'une légère baisse de température.

D'après les conditions climatiques du moment ce mouvement d'eau a vraisemblablement été déclenché par d'importantes chutes de neige fondante sur la partie amont du bassin de la Loire.

En ce qui concerne la température de l'eau, peu de variations ont été notées jusqu'au début de la décrue, le 14 mai (température stable autour de $12^{\circ} \mathrm{C}$ ). Elle a ensuite subi une nette hausse pour se maintenir entre 15 et $25^{\circ} \mathrm{C}$ jusqu'à la fin des opérations (cf. Figure 2).

\section{Synopsis des principaux mouvements observés}

Une ałose a régurgité dès sa remise à l'eau. Les 10 autres ont pu être suivies durant une à sept semaines soit jusqu'à extinction de l'émetteur, soit jusqu'à sortie définitive du tronçon de Loire facilement couvert par repérage routier $(80 \mathrm{~km}$ dont 10 en amont du seuil). Leur comportement migratoire est résumé ci-dessous.

\section{Alose no 1 :}

Ce poisson a d'abord dévalé sur $23 \mathrm{~km}$, puis sa progression vers le seuil s'est déroulée en deux temps, avec un arrêt intermédiaire de deux semaines et une étape finale de $22 \mathrm{~km}$ parcourue en une journée. N'ayant pas franchi l'obstacle malgré 10 jours d'approches répétées, il s'est définitivement replié vers une zone de repos située environ $2 \mathrm{~km}$ en aval.

\section{Alose n' 2 :}

Malgré une reprise de migration immédiate, celle-ci n'a atteint le seuil qu'un mois après son marquage. Elle s'est en effet arrêtée une semaine à l'intérieur d'un pool de repos, avant de dévaler sur au moins $50 \mathrm{~km}$ avec la crue. Elle s'est ensuite comportée comme la précédente (montaison rapide, approche de l'obstacle, repli prolongé). 


\section{Alose no 3 :}

Après quelques déplacements irréguliers celle-ci a amorcé une dévalaison de $300 \mathrm{~km}$ au moment de la montée des eaux. Elle fut recapturée en estuaire,dix jours plus tard.

\section{Alose n०4:}

Marquée un jour avant la crue, sa rapide progression vers l'amont a été stoppée, puis inversée. avant qu'elle n'atteigne le seuil. Reprenant sa migration en même temps que l'alose n० 1 , elle a pu franchir l'obstacle le 19 mai, à l'issue d'une phase d'approche active s'étalant sur moins de 24 heures. II s'agit du seul sujet qui ait réussi à passer le seuil.

\section{Alose no 5 :}

Cet individu équipé en période de crue n'a pas supporté la manipulation. Il a été récupéré mort à l'issue d'une dévalaison progressive.

\section{Aloses nos 6, 7, 8, 9 et 10 :}

Marquées durant une période hydrologique plus favorable, les cinq dernières aloses se sont comportées dé façon analogue. Elles ont rapidement récupéré après leur lâcher (dévalaison sur au plus $8 \mathrm{~km}$ suivi d'une attente inférieure à 24 heures en zone de repos). Leur remontée vers le seuil a ensuite été directe (sans arrêt marqué) et leurs investigations devant l'obstacle semblables à celles des 2 premiers sujets (une ou plusieurs approches actives suivies d'un repli prolongé aux abords d'une zone de regroupement). 

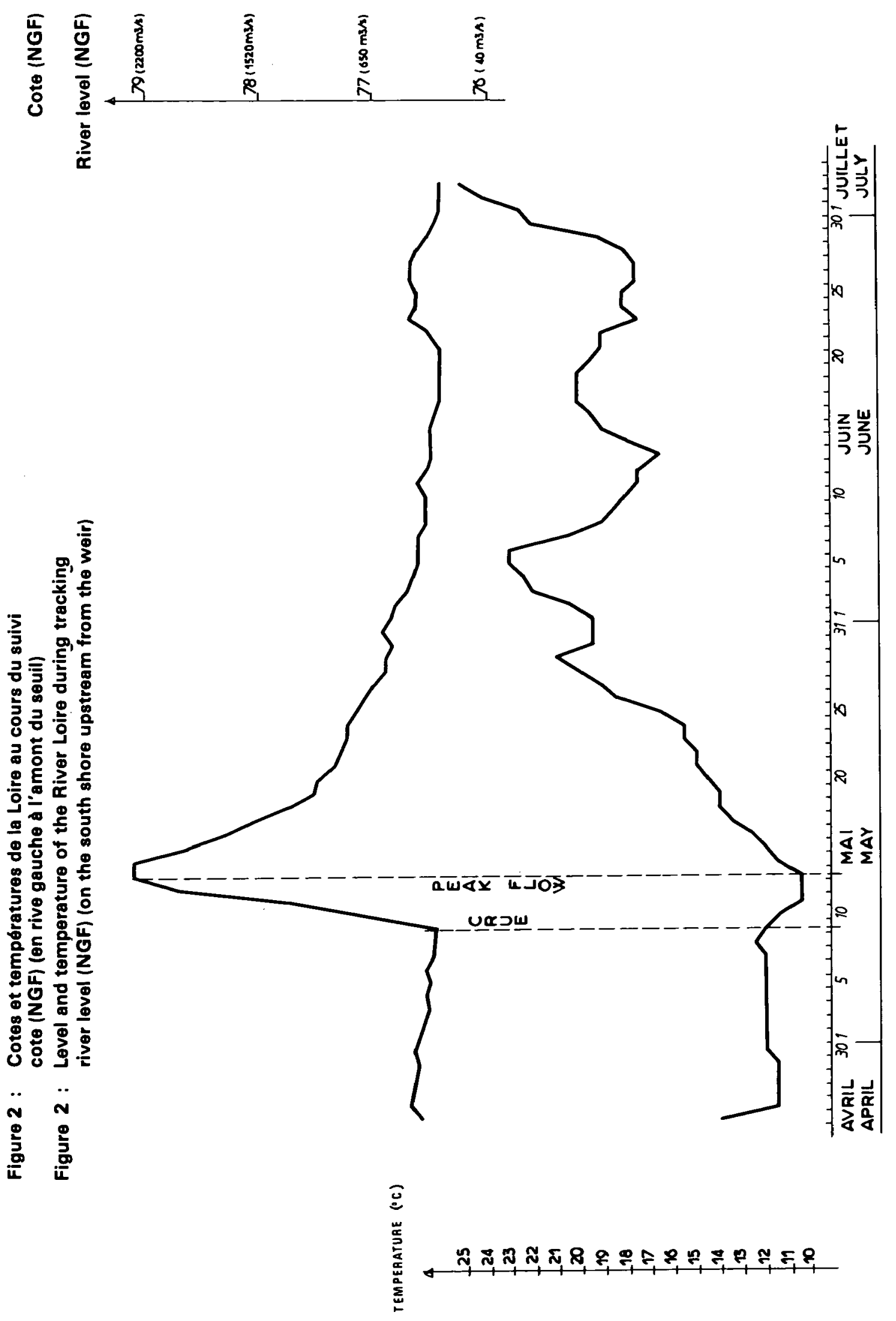


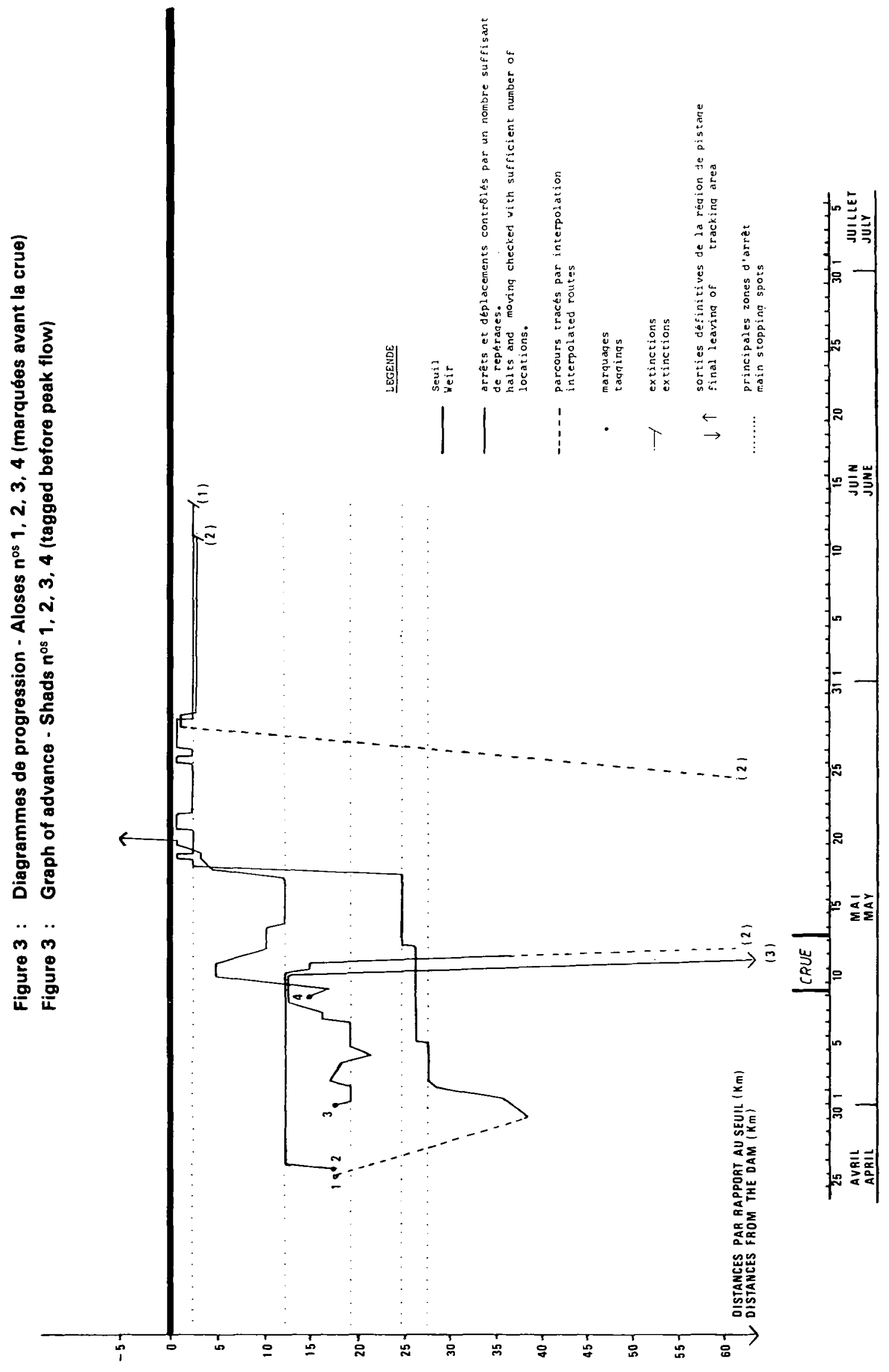




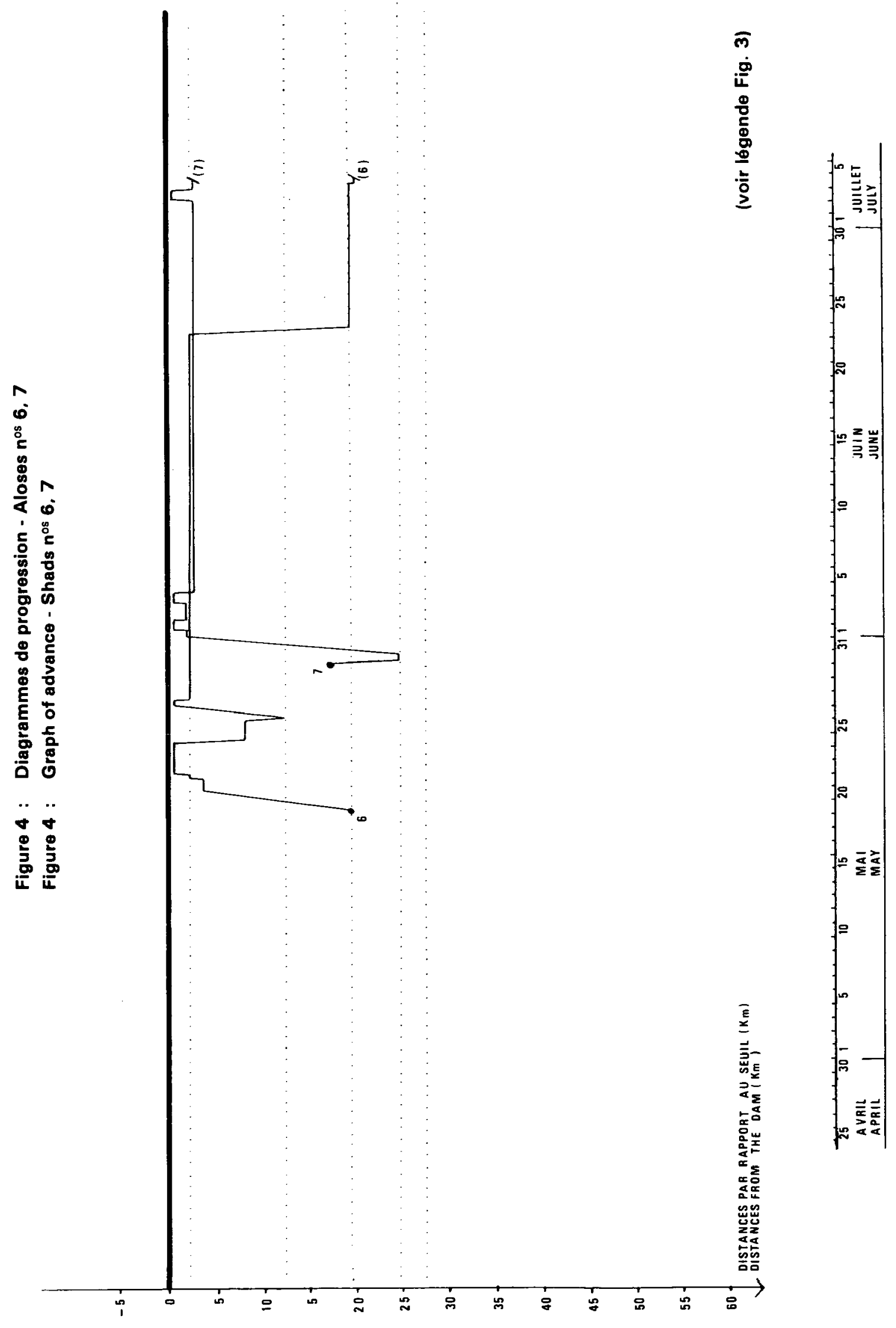


Bull. Fr. Péche Piscic. (1986) 302 : $106-117-114-$

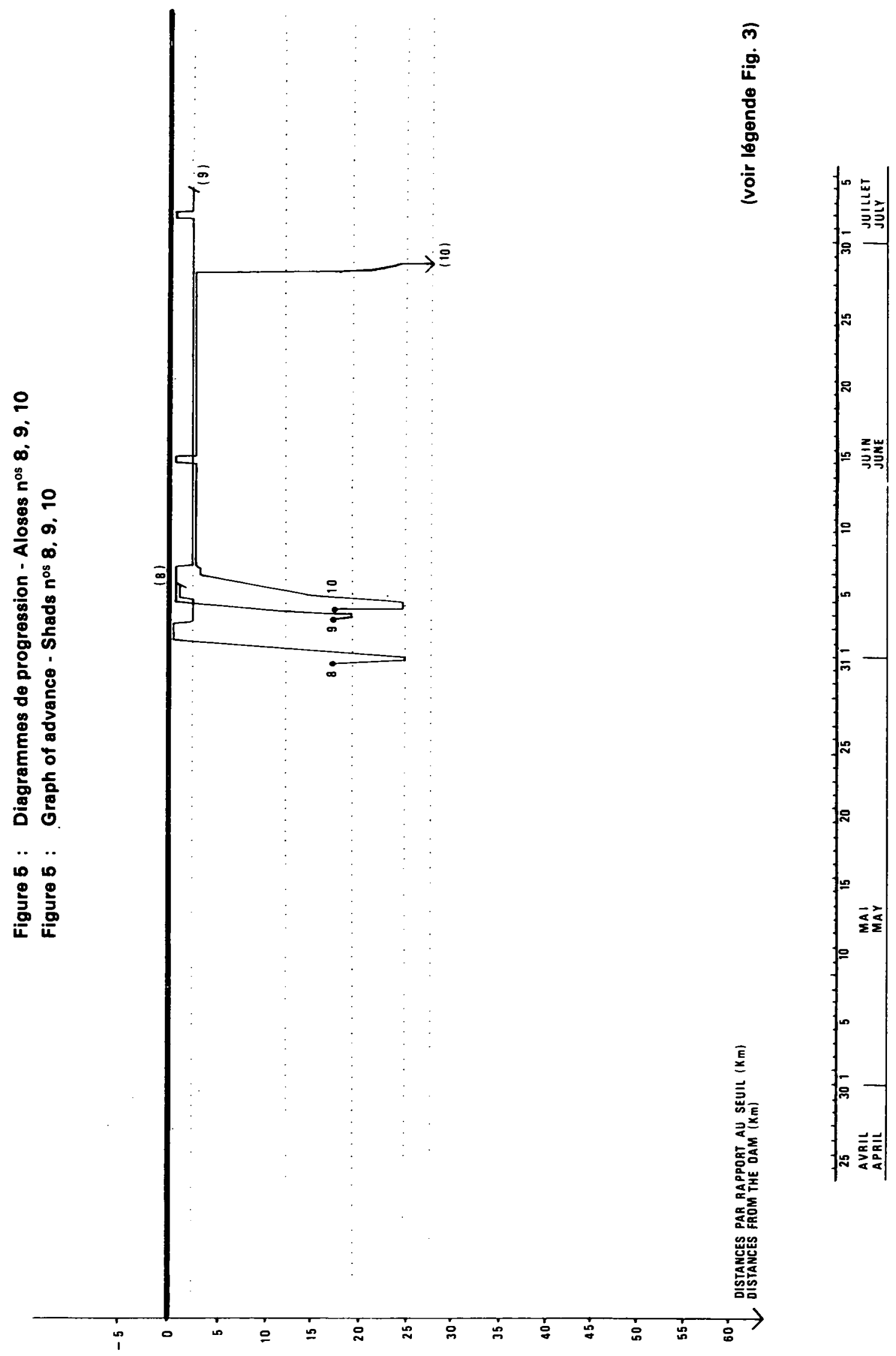




\section{Comportement en aval de l'obstacle}

Le séjour des áloses bloquées à la hauteur de St LAURENT-des-EAUX s'est partagé entre des phases d'approche du seuil et des phases d'attente dans un pool de repli plus d'un kilomètre en aval de l'ouvrage. En moyenne, les poissons se sont présentés deux fois devant l'obstacle, pour évoluer 22 heures consécutives, à l'intérieur de la zone d'influence de celui-ci (jusqu'à $500 \mathrm{~m}$ en aval).

Ces phases d'approche active ont surtout eu lieu durant les premiers jours de présence des aloses sur le site. Deux sont cependant survenues simultanément lors d'un réchauffement des eaux, après 29 jours d'attente pour l'alose $n^{\circ} 7$ et 26 jours pour l'alose $n^{\circ} 9$ (cf. figures 2, 4 et 5).

En ce qui concerne les phases d'attente à l'intérieur de leur pool de repli les poissons se sont limités à quelques déplacements journaliers autour d'une zone de regroupement privilégiée 1,8 km en aval de l'obstacle.

Les aloses marquées ayant atteint St LAURENT-des-EAUX durant une période de décrue progressive, certains changements concernant la localisation de leurs évolutions au pied de l'ouvrage ont pu être constatés. Jusqu'à la fin du mois de mai (débit supérieur à $530 \mathrm{~m}^{3} / \mathrm{s}$ environ) elles ont préférentiellement choisi la proximité de la rive droite lors de leurs investigations devant l'obstacle. C'est d'ailleurs de ce côté que l'alose no 4 a franchi le seuil. Par contre à partir du mois de juin (débit inférieur a $530 \mathrm{~m}^{3} / \mathrm{s}$ environ) les aloses marquées ont nettement privilégié la partie rive gauche de la zone d'influence de l'ouvrage. Ces observations, qui mériteraient confirmation à travers la mise en œuvre de moyens de repérage plus précis, sont à rapprocher de l'évolution que subissent les conditions d'hydraulicité à l'aval du seuil lors des fluctuations du débit de la Loire. Le seuil étant calé à un niveau inférieur en rive gauche sur un tiers de sa longueur la répartition du débit en aval de la chute est très variable. Ainsi pour de faibles hauteurs d'eau au-dessus du seuil, le comportement d'approche des aloses se trouve influencé par l'attractivité accrue de la large veine d'eau qui s'écoule en rive gauche.

\section{Remarque :}

La production de la centrale de St LAURENT-des-EAUX étant en grande partie suspendue durant le suivi des aloses, son fonctionnement $n$ 'a pu influencer les comportements observés (rejet thermique négligeable).

Malgré la faible importance des moyens mis en œuvre (pas de triangulation), les deux traits de comportement qui ont pu être observés en aval du seuil (liaison entre obstacle et pool de repli orientation des approches) mettent en évidence l'intérêt que peuvent présenter de telles investigations (délimitation d'une zone de réserve - positionnement et conception d'un dispositif de franchissement).

\section{DISCUSSION}

\section{Comportement migratoire de la grande alose en Loire moyenne :}

Influence de la température :

Le caractère déterminant de ce facteur sur la migration des aloses a souvent été mis en évidence. Plusieurs études se rapportant à l'espèce américaine (Alosa sapidissima) ont établi qu'il conditionne en grande partie les principales étapes du retour vers les frayères (LEGGETT et WHITNEY, 1972 ; LEGGETT et CARSCADOEN, 1978).

Le présent travail confirme que pour des températures excédant $13^{\circ} \mathrm{C}$ la grande alose est capable de montaison à vitesse soutenue (moyenne des vitesses de migration observées sur la période correspondante $0,85 \mathrm{~km} / \mathrm{h}$ *, soit $20,5 \mathrm{~km} / \mathrm{j}$ ). Par contre, notons que pour des températures égales ou légèrement inférieures à $12^{\circ} \mathrm{C}$, les poissons pistés ont été sujets à des arrêts plus fréquents et surtout nettement plus longs. Rien ne peut prouver que les individus équipés d'émetteurs ne soient pas plus sensibles aux effets limitants de la température à cause du stress qu'ils ont pu subir. Cependant les résultats obtenus s'écartent peu des observations faites par RAMEYE (1976) sur le Rhône et BOISNEAU (1984) sur la Loire, à partir de suivis d'efficacité de pêche sur des poissons non marqués (déplacements faibles ou nuls au-desșous de $11^{\circ} \mathrm{C}$ ).

La détermination d'un seuil fixe de température reste délicate car d'autres éléments peuvent intervenir simultanément: d'une part, l'évolution même du facteur température, d'autre part les fluctuations d'hydraulicité.

\footnotetext{
* Hors phase de récupération consécutive au marquage et d'attente en aval du seuil.
} 


\section{Influence d'une forte crue :}

Au vu des déplacements anadromes qu'ont réalisés les quatre premières aloses, avant comme aprés le passage de la crue, le mouvement général de dévalaison constaté les 10 et 11 mai peut sans nul doute être attribué à la brusque montée des eaux qui eut lieu les mêmes jours (cf. figure $n^{\circ} 3$ ). 11 est probable que plusieurs facteurs tels que le refroidissement, la baisse de la teneur en oxygène dissous, l'accélération du courant, le charriage ou l'excès de turbidité, interviennent dans l'explication de ce comportement.

Dans de telles conditions les aloses suspendraient leur progression anadrome comme certains pêcheurs ont pu le constater sur le Rhône lors des crues de l'Isère (RAMEYE et al., 1976). D'après le résultat des pistages réalisés en Loire pendant la crue de mai 1985, ces migrateurs auraient même tendance à dévaler sur des distances parfois importantes, s'arrêtant en rive dans des zones de repos caractéristiques (connues par les pêcheurs de Loire sous le nom de "bouge").

A l'inverse, les aloses trouveraient en période de décrue un environnement stimulateur. progressant à des vitesses pouvant atteindre $2 \mathrm{~km} / \mathrm{h}$ * contre un courant encore rapide (localement $7 \mathrm{~km} / \mathrm{h}$ dans le chenal).

\section{Fiabilite technique :}

\section{Marquage :}

Le présent travail montre que, moyennant certaines précautions, la grande alose peut supporter l'insertion par voie buccale d'un émetteur sans manifester de gêne apparente si ce n'est au travers d'une courte phase de dévalaison après son marquage. Plusieurs observations tendent à prouver que les poissons surmontent le stress provoqué par leur manipulation:

- ils rétablissent rapidement leur équilibre dans le bac de marquage et manifestent une vivacité rassurante au moment de leur lâcher

- certains d'entre eux, peu traumatisés, reprennent leur progression anadrome sans dévalaison préalable, après quelques heures de récupération seulement

- ils sont capables d'efforts de nage soutenus (franchissement d'un obstacle marqué pour l'alose $n^{\circ} 4$ et montaison rapide pour les aloses nos $1,4,6,7,8,9$ )

- leurs mouvements peuvent être suivis sur une longue période et la mobilité dont ils font preuve plus d'un mois après leur marquage, témoigne de leur possibilité d'adaptation à l'ingestion partielle d'un émetteur (aloses nos 7 et 9 en particulier).

Deux limitations peuvent cependant compromettre la réussite de l'opération :

- une alose soumise à des conditions défavorables lors de sa remise à l'eau (crue ou basse température) risque de dévaler sur des distances importantes et de retarder sa reprise de migration

- parmi les sujets capturés l'élimination des moins résistants vis-à-vis du stress de manipulation reste aléatoire.

\section{Pistage :}

Les moyens mis en cuvre pour le suivi conditionnent la réussite des opérations au même titre que le marquage lui-même. Leur diversification (voie routière pour la rapidité d'exécution, fluviale pour la précision, et aérienne pour l'extension géographique) permet de réaliser un pistage complet des aloses, qui se sont révélées être mobiles de jour comme de nuit, non seulement à l'échelle de leurs déplacements migratoires, mais aussi durant leurs arrêts de migration.

De plus, la fiabilité des émetteurs utilisés est confirmée : puissance d'émission suffisante et homogène, bonne stabilité en fréquence, durée de vie conforme aux indications. (Ce matériel étant sûr il est notamment possible d'exploiter l'information négative obtenue à l'issue de recherches vaines sur un tronçon déterminé).

\section{CONCLUSION}

Bien que l'alose soit un poisson délicat à manipuler, l'essai de radio-pistage que nous avons tenté en Loire sur Alosa alosa s'avère concluant. Cette application ouvre certaines perspectives intéressantes: dans le cadre d'investigations à plus grande échelle, elle pourrait notamment contribuer à la localisation des frayères dans un bassin comme celui de la Loire, où le parcours de ce grand migrateur est mal connu ; par ailleurs, dans le but de concevoir, d'améliorer ou de tester un dispositif de franchissement, la mise en place de systèmes de détection et de repérage précis permettrait l'étude détaillée du comportement d'approche de ce poisson réputé méfiant.

L'intérêt de telles recherches porte sur la préservation et la restauration du potentiel reproducteur de la grande alose au sein de nos systèmes fluviaux.

\footnotetext{
* Vitesses moyennes relevées sur des distances avoisinant $10 \mathrm{~km}$.
} 


\section{BIBLIOGRAPHIE}

BOISNEAU P., 1984. Etude préliminaire de la biologie de la grande alose (Alosa alosa Linné, 1758) de la Loire : activité de migration, biométrie et maturité sexuelle. D.E.A. "Sciences et Techniques de l'Environnement". Université de Paris Val-de-Marne. Ecole Nationale des Ponts et Chaussées. Conseil Supérieur de la Pêche, Poitiers. 34 p.

CASSOU-LEINS, CASSOU-LEINS, 1981. Recherche sur la biologie et l'halieutique des migrateurs de la Garonne et principalement de l'alose, Alosa alosa Linné. Thèse de 3ème cycle. Sci. Techn. Prod. Anim., Inst. Nat. Polytechnique, Toulouse. 392 p.

C.T.G.R.E.F., 1975. Les obstacles à la migration des poissons du Rhône dans le département du Gard. Division Q.E.P.P. Group. Antony. Etude n० 8, 54 p. + annexes.

DAUTREY L., LARTIGUE P., 1983. Recherche sur la migration des aloses (Alosa alosa) et des truites de mer (Salmo trutta) en Garonne (Site de Golfech). Thèse de 3ème cycle. Inst. Nat. Polytechnique, Toulouse. $212 \mathrm{p}$.

DODSON J.J., LEGGETT W.C., 1972. The behavior of adult American shad (Alosa sapidissima) during migration from salt to fresh water as observed by ultrasonic tracking techniques. J. Fish. Res. Board. Can., 29 : 1445-1449.

DODSON J.J., LEGGETT W.C., 1973. Behavior of American shad (Alosa sapidissima) homing to the Connecticut River from Long Island Sound. J. Fish Res. Board Can., 30: 1847-1860.

DODSON J.J., LEGGETT W.C., 1974. Role of olfaction and vision in the behavior of American shad (Alosa sapidissima) homing to the Connecticut River from Long Island Sound. J. Fish Res. Board Can., 31 : 1607-1619.

DOUCHEMENT C., 1981. Les aloses des fleuves français Alosa fallax Lacépède et Alosa alosa Linné. Biométrie. Ecobiologie. Autonomie des populations. Thèse 3ème cycle. Ecol. Gen. App., Montpellier I, 393 p. + Bibl.

HYDRO-QUEBEC, 1982. Etude télémétrique du comportement migratoire de l'alose savoureuse. Centrale Rivière des Prairies. Remplacement de l'évacuateur de crue et arasement d'un haut fond. Rapport complémentaire, $\mathbf{5 8}$ p. + annexes.

LECLERCA., 1941. Note sur des essais de reproduction artificielle de l'alose dans le bassin de la Loire. Bull. Fr. Pisc., $123: 27-37$.

LEGGETT W.C., CARSCADDEN J.E., 1978. Latitudinal variation in reproductive characteristics of American shad (A/osa sapidissima) : evidence for population specific life history strategies in fish. J. Fish Res. Board Can., 35 : 1469-1478.

LEGGETT W.C.. JONES R.A., 1971. Net avoidance behavior in American shad (Alosa sapidissima) as observed by ultrasonic tracking techniques. J. Fish Res. Board Can., 28 : 1167-1171.

LEGGETT W.C., WHITNEY R.R., 1972. Water temperature and the migrations of American shad. Fish Bull., 70:659-670.

MENNESSON C., 1984. Etude préliminaire de la biologie de la grande alose (Alosa alosa Linné, 1758) de la Loire : activité de migration, scalimétrie et otolimétrie. D.E.A. Eco-Ethologie. Université Rennes I. Conseil Supérieur de la Pêche, Poitiers, $59 \mathrm{p}$.

RAMEYE L., KIENER A., SPILLMANN C.J., BIOUSSE J., 1976. Aspects de la biologie de l'alose du Rhône. Pêche et difficultés croissantes de ses migrations. Bull. Fr. Pisc. $263: 50-76$.

SPILLMANN C.J., 1961. Faune de France. 65 poissons d'eau douce. Le Chevallier, Paris, 303 p. 